\title{
Lenguaje Del Diseño
}

\author{
LANGUAGE OF DESIGN
}

Mg. Pablo Vélez Ibarra

Universidad Tecnológica Israel

pvelez@uisrael.edu.ec

Fecha de recepción: 18/05/2014

Fecha de aceptación: 16/06/2014

\section{Resumen}

El diseño gráfico es un marco bastante amplio dentro de la comunicación visual y que permite englobar una serie de actividades humanas que buscan, en primera instancia, transmitir una serie de elementos comunicacionales, transformados en productos tangibles. Para llegar a esta instancia final, se necesita pasar por un proceso de realización o creación y parte inicial de este proceso es el lenguaje del diseño gráfico, el que se nutre de algunos componentes teóricos y prácticos. En este sentido serán motivo de estudio la Forma y el Espacio; el espacio positivo y negativo y la composición. Claro está que se debe indicar que este texto busca enfocarse en estos elementos como un conjunto de herramientas dentro del diseño gráfico y la comunicación visual.

Palabras clave: Diseño gráfico, lenguaje del diseño gráfico, espacio, espacio positivo-negativo, composición. 


\section{Abstract}

Graphic design is a wide field within the world of visual communications that allow us to incorporate a series of human activities that seek, in the first place, to communicate ideas that can be transformed into tangible products. To reach that final stage, it must pass through a process of creation where the initial part of the process is the language of the graphic design, which in itself feeds from theoretical and practical components. In that context Form and Space will be the fact of the matter; positive or negative Space and the Ensemble of the whole. This article focuses on those elements as a set of tools within graphic design and visual communication.

Keywords: Graphic design, language of graphic design, form, space, positive-negative space, composition. 


\section{Introducción}

Con el pasar del tiempo, las distintas sociedades han utilizado distintos tipos de lenguajes para comunicarse. Estos lenguajes han ido cambiando o evolucionando, ya sea porque un pueblo grande o más poderoso (militar, cultural o de cualquier otra índole) ha conquistado este pueblo, ya sea porque un pueblo guerrero y con una cultura distinta ha dominado a otra, como en el caso de los griegos o romanos, o porque un pueblo ha realizado un proceso de aculturación o también “...un cambio cultural empieza con el proceso de innovación, la formación de un nuevo hábito por un solo individuo y que subsecuentemente es aceptado o aprendido por otros miembros de su sociedad" (Murdok s/a, pág. 4)

El tema de la comunicación es apasionante. Sin embargo, se busca alcanzar un punto medio de discusión o reflexión entre la comunicación y el diseño gráfico. Si volvemos al lenguaje del diseño gráfico es importante anotar que:

"si se hace referencia a la producción de mensajes en lenguaje gráfico, es preciso considerar que cada elemento que se escoja para la composición está cargado de un alto potencial significativo desde el punto de vista visual y que, manejado adecuadamente, llega a constituir una sólida base comunicación del mensaje. Si en cambio no es manejado de manera adecuada, producirá resignificaciones en el público que no son las deseadas, o incluso las menos convenientes a nuestros objetivos comunicacionales" (Boscardin, pág. 1)

Por otro lado para plasmar "ese algo" -la idea que ronda en nuestra mente o en las mentes del equipo de trabajo-, es necesario utilizar herramientas que permitan materializar una idea, por más simple, sencilla, disparatada o ridícula que esta sea. Como escribió Gerard Piel: "sin la publicación la ciencia está muerta" (Day, 1996), de la misma manera si no se plasma la idea sobre algún soporte, ésta no existe.

"Siempre que se diseña algo, o se hace, bocetea y pinta, dibuja, garabatea, construye, esculpe o gesticula, la sustancia visual de la obra se extrae de una lista básica de elementos. Y no hay que confundir los elementos visuales con los materiales de un medio, como la madera, el yeso, la pintura o la película plástica.

Los elementos visuales constituyen la sustancia básica de lo que vemos y su número es reducido: punto, línea, contorno, dirección, tono, color, textura, dimensión, escala y movimiento. Aunque sean pocos, son la materia prima de 
toda la información visual que está formada por elecciones y combinaciones selectivas. La estructura del trabajo visual es la fuerza que determina qué elementos visuales están presentes y con qué énfasis." (Dondis, 2007).

Con los elementos antes mencionados se logra una comunicación muy fluida, pero hay que tomar en cuenta que para trabajar con cada uno de estos elementos necesitamos bastante tiempo para conocerlos bien y ponerlos en práctica. Sin embargo, por tratarse de un artículo, se revisarán algunos elementos como la forma, el espacio y la composición.

\section{Lenguaje del diseño gráfico}

\section{¿A qué nos referimos con lenguaje del diseño gráfico?}

En primer lugar, el lenguaje hace relación con la "capacidad propia del ser humano para expresar pensamientos y sentimientos por medio de un sistema de signos orales y escritos..." (Diccionario Manual de la Lengua Española Vox, 2007); en segundo lugar, diseño gráfico es un término joven que aparece en 1922 en Estados Unidos con W. A. Dwiggins, y que podría significar "...la transformación de ideas y conceptos en una forma de ordenar estructural y visual. Es el arte de hacer libros y revistas, anuncios, envases o folletos" (Hermann Blume, 1988). Sin embargo para mi criterio diseñar es realizar un estudio que permita el análisis para solucionar una determinada necesidad o posible problema. Bajo este criterio se puede decir que el lenguaje del diseño es "la capacidad del ser humano para expresar mediante elementos visuales, posibles respuestas a necesidades comunicacionales por medio de productos tangibles e intangibles".

Para Jorge Frascara (2004), "el diseño gráfico es fundamentalmente comunicación humana, y el trabajo del diseñador es construir una estructura virtual, algo similar a una partitura musical, con el fin de organizar un evento que tiene lugar cuando un observador se encuentra ante un diseño".

Este artículo sobre diseño gráfico, recuerda que esta especialidad debe ser vista como un medio de comunicación que permita facilitar las diversas actividades para conseguir una serie de productos comunicacionales, los mismos que se verán más adelante.

Dentro del proceso del lenguaje del diseño gráfico es importante recordar que el ser humano realiza una "interpretación que los ojos y el cerebro hace de lo que vemos [...] seamos conscientes o no, los ojos proporcionan información constante al cerebro, que procesa e interpreta los datos visuales que recibe." (Dabner, 2005).

Finalmente, se puede decir que el lenguaje del diseño gráfico sumado a la comunicación, son actividades humanas que se desarrollan para generar algún modelo comunicacional visual "con el propósito de afectar el conocimiento, las actitudes y el comportamiento de la gente". (Frascara, 2004). 


\section{Forma y espacio}

Al llegar a este punto y darnos cuenta del mundo que nos rodea, podemos encontrar infinitas formas dentro del espacio circundante. Un concepto importante a definir es la forma, que según el diccionario de la lengua española es la "configuración externa de algo". Por otro lado la forma se encuentra representada sobre un lugar. Este lugar también podría ser llamado espacio y sería un lugar tangible o intangible en el que se encuentra representado una forma, que tendría características particulares como son su tamaño, textura, color, peso e incluso su función. La forma tiene y debe estar en un espacio determinado.

Este concepto puede ser real o irreal, dependiendo del contexto en el que se lo interprete (pero que funciona bien en este contexto); y, a que me quiero referir con espacio real, pues es ese lugar que posee como mínimo alto y ancho, es decir, dos dimensiones. Sin embargo, nuestro espacio físico tiene una dimensión más, la profundidad. Por otro lado, también está el espacio irreal, el cual puede ir más allá de las nociones reales y físicas de lo que conocemos como tangible y que puede funcionar con cuatro, cinco o más dimensiones -será esto irreal- en fin, solamente nos quedaremos en esta oportunidad con el estudio de las dos dimensiones aplicadas a la composición de elementos.

Es importante mencionar que algunos ejemplos que se mostrarán en este artículo han sido tomados del libro de David Dabner "Diseño Gráfico. Fundamentos y Prácticas". Es ese sentido empezaremos nuestro estudio analizando lo que puede ocurrir dentro de un cuadro (bueno, esto se lo puede realizar dentro de cualquier figura, pero para analizar conceptos es mejor la figura geométrica que se muestra a continuación).

Gráfico $N^{\circ}$ 1: Relaciones entre las formas.
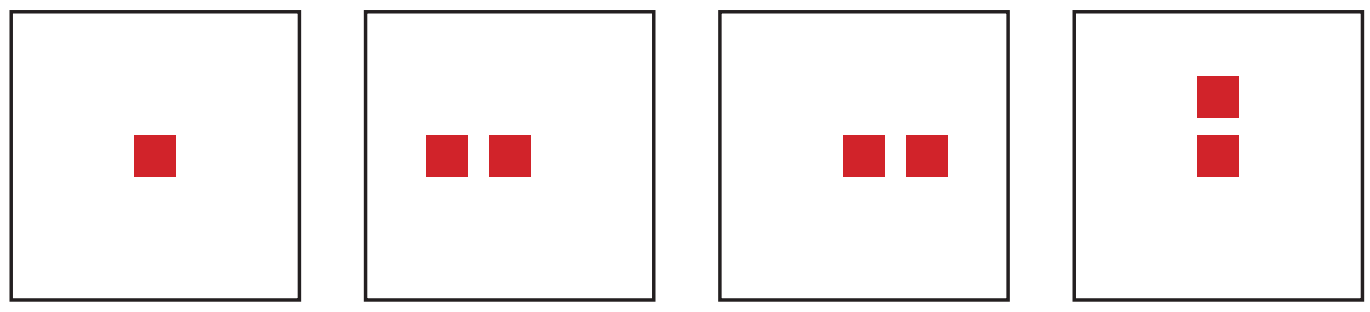

Tomado de: Diseño Gráfico, Fundamentos y Prácticas de David Dabner

En primera instancia (letra a) tenemos un cuadrado en el centro de otro cuadrado, como se podrá observar este primer conjunto se encuentra estático o pasivo, ya que el espacio que lo rodea es igual por los cuatro lados. 
En segunda instancia introducen dos cuadrados por la izquierda (letra b), es aquí donde se generan fuerzas visuales y al parecer tendríamos movimiento. Posteriormente, en la tercera instancia (letra c) la posición de los cuadrados sugiere un movimiento hacia la derecha y finalmente la posición de los cuadrados insinúa un movimiento descendente (letra d).

Después del ejemplo anterior, es importante pensar en el proceso de comunicación que se genera con formas sencillas, qué es lo que puede transmitir y qué es lo que se puede interpretar, ya que aquí también entra en función el acervo cultural de la persona que observa las distintas relaciones entre formas. Este mismo ejemplo se lo podría realizar con letras, con imágenes, o cualquier elemento visual que el estudiante desee utilizar. Esta pequeña reflexión que la mayoría conoce consciente o inconscientemente, sirve como hilo conductor para hablar sobre fondo y figura. Para Dabner (2005) una forma siempre se percibe en relación con el espacio en que se enmarca y con las demás formas presentes en éste. A continuación se analizará el siguiente gráfico:

Gráfico $\mathrm{N}^{\mathrm{o}}$ 2: Fondo y figura.

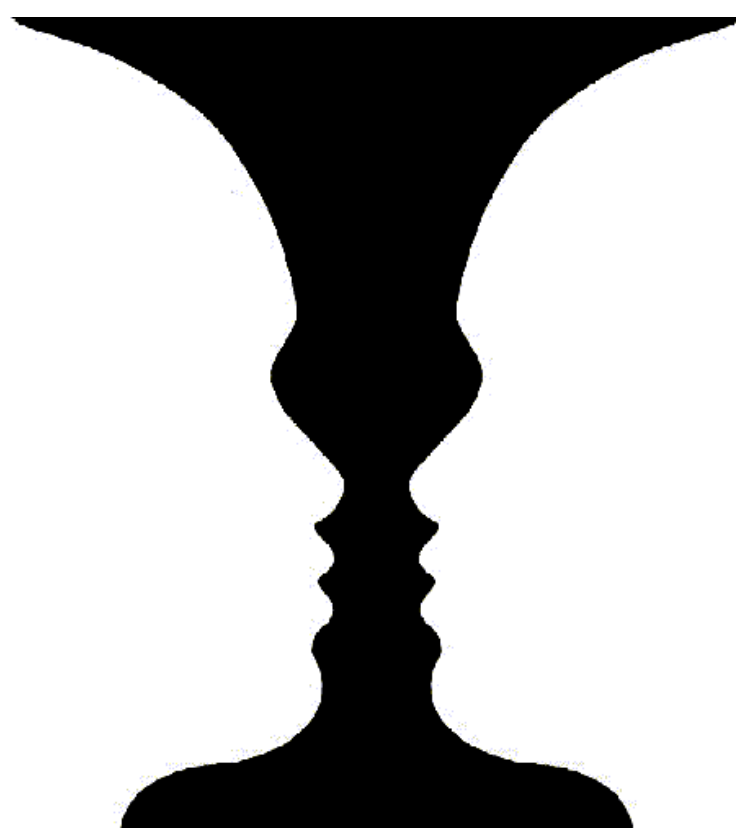

Tomado de: http://gestaltvenezuela.blogspot.com/

Aquí se tiene un ejemplo interesante y que ha sido utilizado ampliamente para analizar el concepto del fondo y la figura. En este sentido -y dependiendo de la persona que lo vea y lo que se quiera entender...- citaré solamente tres aspectos de esta imagen. 
Primero tenemos la presencia de una copa o copón, segundo tenemos dos rostros que se miran frente a frente y como tercero una simple mancha. Usted estimado lector sabrá cuál elegir. Sin embargo, esta es la muestra más simple de fondo y figura, el mismo que ha sido ampliamente desarrollado y discutido por la Gestalt.

La teoría de la Gestalt se refiere a las estrategias usadas por el sistema perceptivo para crear todos coherentes interpretables. La función organizativa del sistema perceptivo está orientada a un fin, y el fin es encontrar significado. Dada la conexión entre percepción, significado y supervivencia, es fácil comprender que tengamos una necesidad emocional de entender lo que vemos. Cuando percibimos sin entender sentimos aburrimiento, desasosiego, fatiga o miedo, según las circunstancias. La percepción no es un acto sin urgencia. Vemos para entender. Necesitamos entender para reaccionar. (Frascara, 1996).

A continuación se muestran otros ejemplos sobre el fondo y la figura:

Gráfico № 3, 4 y 5: Ejemplos de fondo y figura.
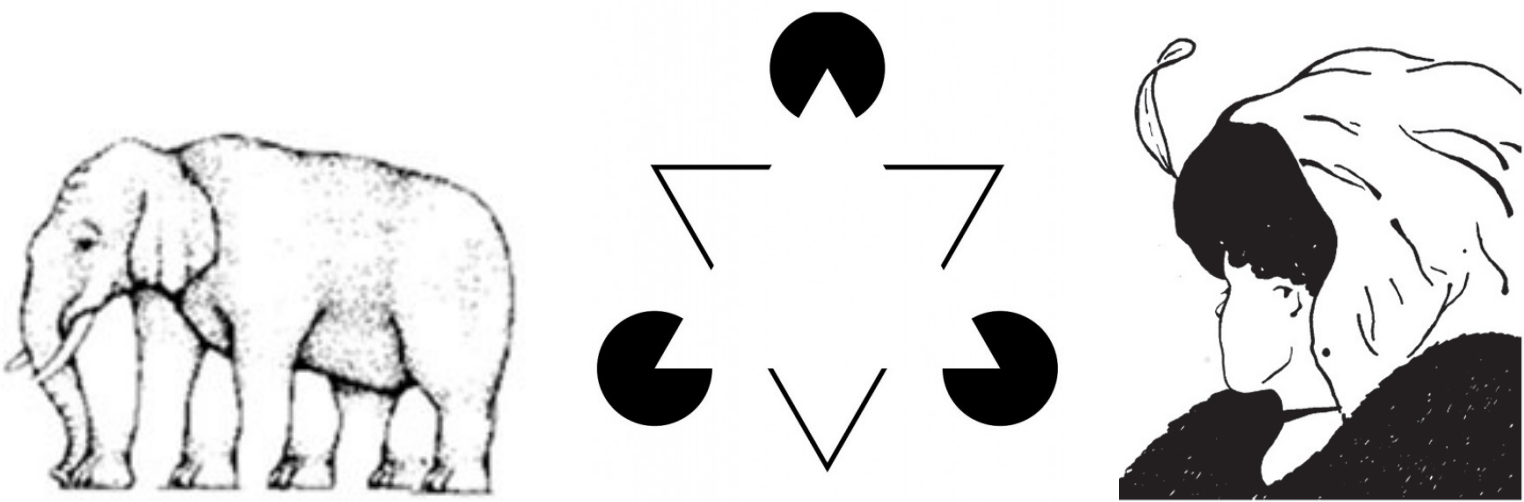

Tomado de: http://guillermoleone.com.ar/leyes.htm

Finalmente y entrando en el contexto de la Gestalt, sería oportuno incluir el significado de una de las leyes o principios de la Gestalt que es la figura y el fondo, que dice:

Figura, es un elemento que existe en un espacio o campo destacándose en su interrelación con otros elementos. Fondo: todo aquello que no es figura. Es la parte del campo que contiene elementos interrelacionados que no son centro de atención. El fondo sostiene y enmarca a la figura y por su contraste menor tiende a no ser percibido o a ser omitido. (Leone, 2013) 


\section{Espacio positivo y negativo}

Todas las formas pertenecen a un contexto del que no pueden aislarse. En general se consideran positivas, y el espacio que las rodea negativo. El espacio contenido en un formato, a veces descrito como el fondo, es un elemento crucial en cualquier diseño y no sólo lo que queda cuando se coloca en él una forma. (Dabner, 2005).

Siguiendo el tema de espacio positivo y negativo, continúa Dabner explicando que:

“...El espacio positivo es aquel que ocupa el área de la imagen, mientras que el espacio negativo es el fondo, también conocido como plano de la imagen. En este sentido, hay que ser consciente de que, en general, el espacio negativo funciona como apoyo al área de la imagen y no se debe permitir que se desarrolle como un espacio activo autónomo" (Ibíd).

\section{Composición}

El tema de la composición no sólo es una palabra. Esta abarca varios conceptos que comprenden varias actividades, y como se ha venido mostrando a lo largo de este artículo, se busca partir de un significado simple o tal vez sencillo, así el Diccionario de la Lengua Española - Vigésima segunda edición dice lo siguiente sobre la composición: "Esc. y Pint. Arte de agrupar las figuras y accesorios para conseguir el mejor efecto, según lo que se haya de representar".

A lo largo de la historia de las artes visuales ha habido distintas teorías de composición. Vitrubio, el arquitecto e ingeniero romano, creó una fórmula matemática para la división del espacio dentro de un cuadro. Su solución conocida como la Sección Áurea, también llamada Regla Áurea, se basaba en una proporción fija entre los lados largos y cortos de un rectángulo. (Dabner, 2005)

La sección áurea se halla presente en la naturaleza, el arte, la arquitectura. Las piñas de los pinos, las conchas marinas y el cuerpo humano, por ejemplo, la respetan. Piet Mondrian y Leonardo Da Vinci, incorporaron la sección áurea a sus pinturas, mientras que Stradivarius la utilizó en la construcción de sus violines. El Partenón, la Gran Pirámide de Gizeh, Stonehenge y la Catedral de Chartres, son otros de los casos en los que se siguió este principio. [...] Tanto si la sección áurea tiene algo que ver con preferencias estéticas inherentes al ser humano como si sólo se tratara de una antigua técnica de diseño convertida en tradición, no hay duda de su influencia pasada y presente en el campo del diseño.

La sección áurea es la proporción entre dos segmentos, de manera que el más pequeño (bc) es el más grande $(\mathrm{ab})$, lo que el más grande (ab) es la suma de los dos segmentos (ac), es decir, bc/ab=ab/ac=0,618 (Lidwell, Holden \& Butler, 2008). 
Otro elemento utilizado para conseguir una buena composición y distribución de los elementos en un determinado espacio es la sucesión de Fibonacci, que fue creada por el matemático italiano Leonardo de Pisa con su obra Liber Abaci en 1202.

Una sucesión de Fibonacci, es una secuencia de números en la que cada uno es la suma de los términos anteriores $(1,1,2,3,5,8,13 \ldots)$. Con frecuencia se observan patrones de esta secuencia en las formas de la naturaleza, como los pétalos de las flores, las espirales de las galaxias y los huesos de la mano humana. La omnipresencia de esta secuencia en la naturaleza ha llevado a la conclusión de que los patrones basado en la sucesión de Fibonacci son intrínsecamente estéticos y, por tanto merecen un puesto importante en el campo del diseño.

Los patrones de Fibonacci están presentes en muchas obras clásicas, tanto poéticas como artísticas, musicales y arquitectónicas. Por ejemplo, algunos estudios de literatura afirman que Virgilio empleó secuencias de Fibonacci para estructurar la métrica de la Eneida. También hallamos sucesiones de Fibonacci en las composiciones musicales de las sonatas de Mozart y en la Quinta sinfonía de Beethoven. Le Corbusier combinó medidas clave del cuerpo humano con sucesiones de Fibonacci para desarrollar el Modulor, un sistema clásico de proporciones y medidas arquitectónicas para ayudar a los diseñadores a conseguir diseños prácticos y armoniosos. (Ibíd).

La sección áurea es un número irracional (un decimal infinito) y se puede computar con la ecuación $(\sqrt{5}-1) / 2$. Si se añade 1 a la sección áurea se obtiene1,618..., el número Phi. Los valores se intercambian para definirla, ya que representan la misma relación geométrica básica. Las formas geométricas derivadas de la sección áurea incluyen elipses rectángulos y triángulos. 
Gráfico $\mathrm{N}^{\circ}$ 7: La sección áurea, aplicaciones.
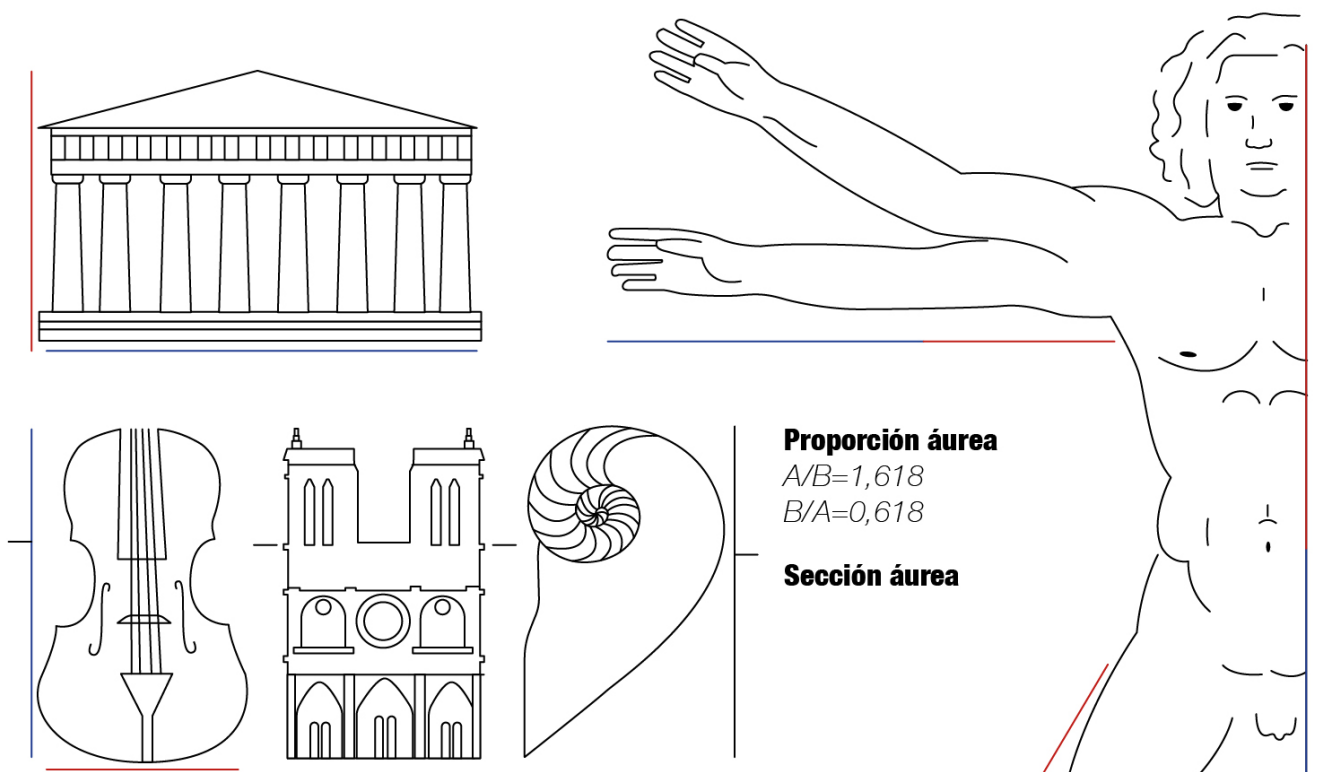

$A / B=1,618$

$B / A=0,618$

Sección àurea
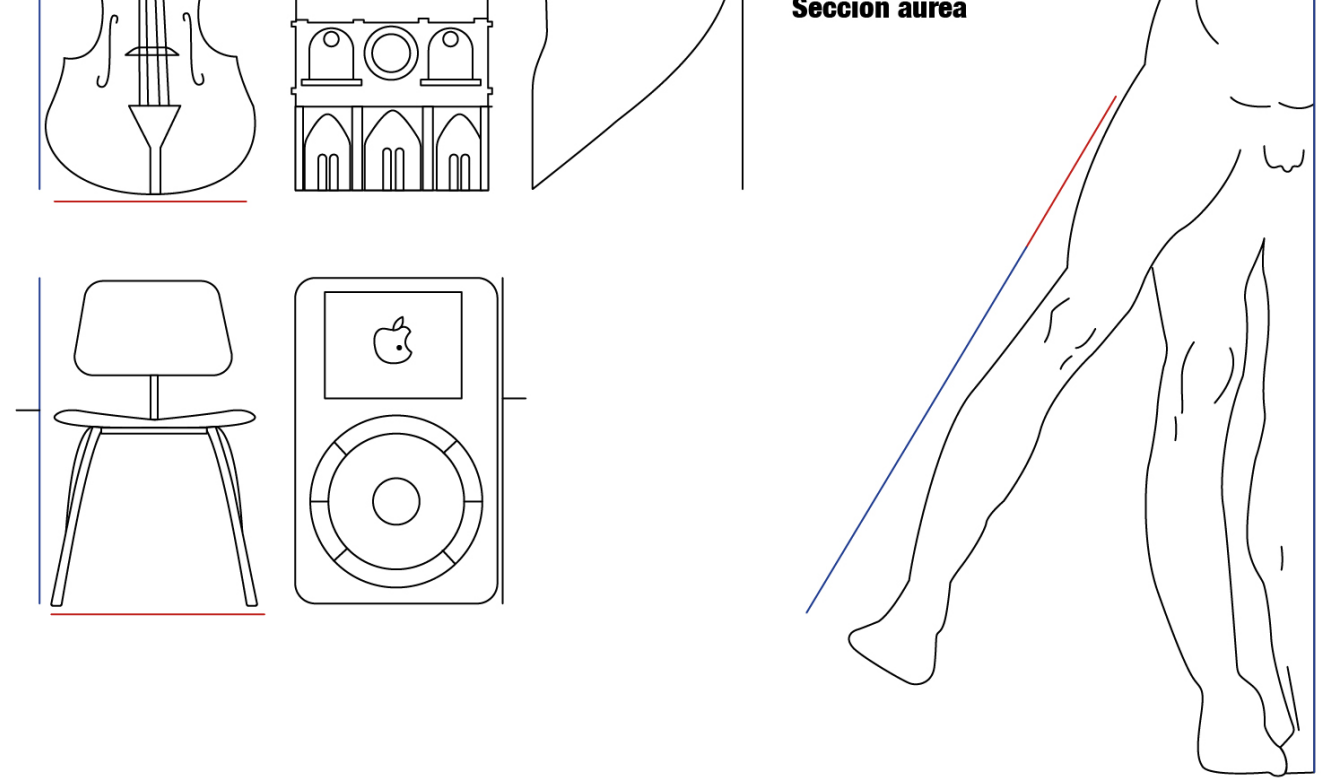

Tomado de: Pricipios universales del diseño. Lidwel, Holden \& Butler. (2008) 
Nota: Dentro de la imagen original el gráfico $\mathrm{n}^{0} 7$ posee un texto que dice lo siguiente:

En cada ejemplo, la proporción entre el segmento azul y el rojo se aproxima a la sección áurea. Observe que la proporción se corresponde con un elemento significativo o bien con una alteración de la forma. Los ejemplos mostrados son: el Partenón, un violín Stradivarius, la Catedral de Notre Dame, una concha de nautilo, una silla Eames, un aparato reproductor MP3 Apple iPod y el hombre Vitrubio de Leonardo Da Vinci.

Gráfico No 8: La sección áurea, aplicaciones.

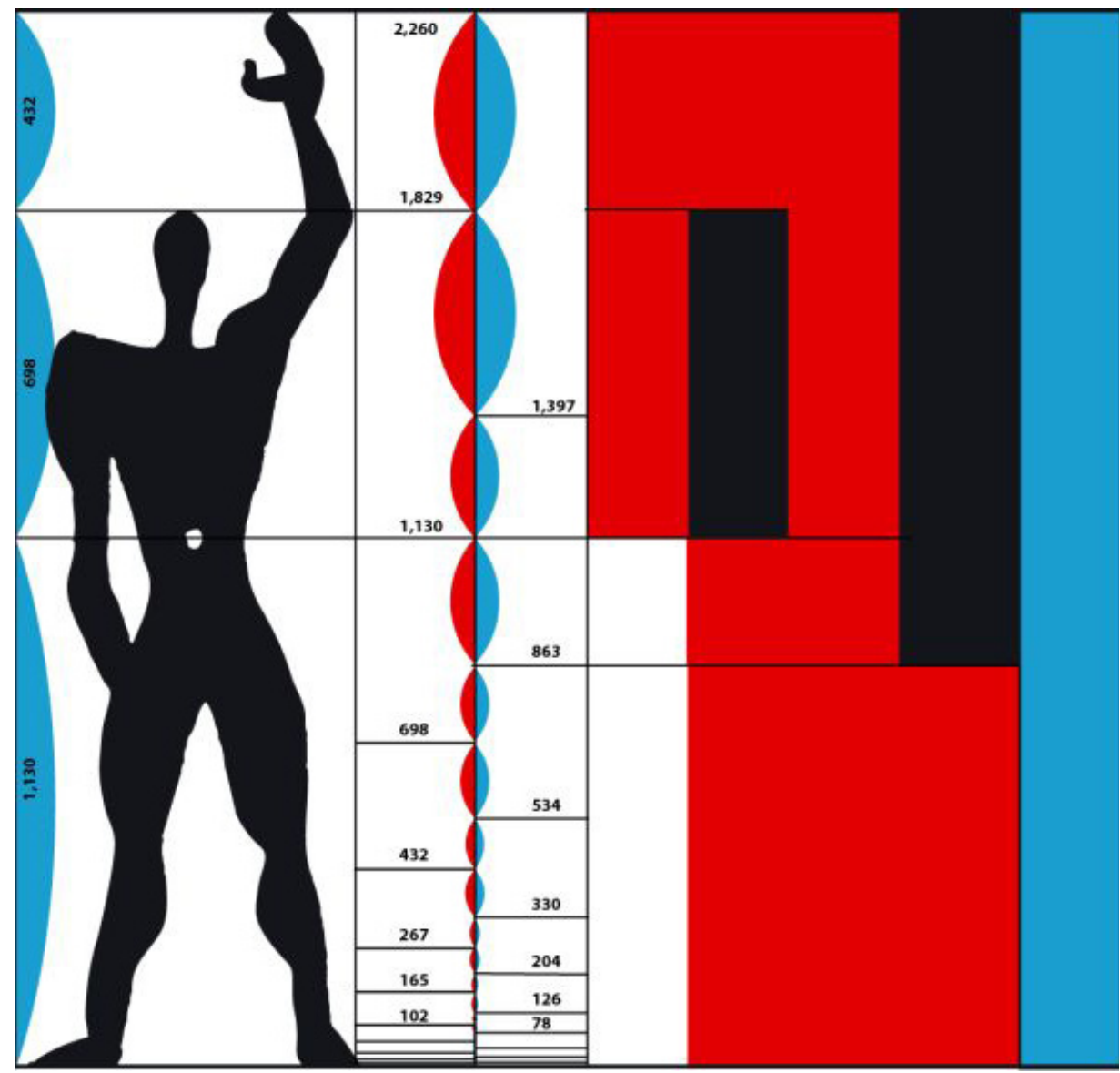

Tomado de: Pricipios universales del diseño. Lidwel, Holden \& Butler. (2008) 
Nota: en la figura original (gráfico $\mathrm{n}^{\circ} 8$ ), hay un texto en la parte superior izquierda que nos indica que:

Le Corbusier derivó dos secciones de Fibonacci basadas en características básicas del cuerpo humano para crear el Modulor. Las secuencias representarían un conjunto de medidas ideales para ayudar a los diseñadores a conseguir una proporciones práctucas y armoniosas. Las secciones áureas se calculaban dividiendo cada número de la secuencia por el número anterior, (indicado por las líneas horizontales). 


\section{Bibliografía}

Blume, H. Haga ud. mismo su diseño gráfico. Hermann Blume Ediciones, S.A. España, Madrid. 1992.

Dabner, D. Diseño gráfico. Fundamentos y prácticas. Blume. Barcelona. 2005

Day, R. Cómo escribir y publicar trabajos científicos. Organización Panamericana de la Salud. Washington D.C. EUA. 1996.

Dondis, D. La sintaxis de la imagen. Introducción al alfabeto visual. GG Diseño. Barcelona, España. 2007.

Diccionario Manual de la Lengua Española Vox. Larousse Editorial, S.L. (C) 2007.

Diccionario de la Lengua Española - Vigésima segunda edición.

Frascara, Jorge. Diseño gráfico para la gente. Comunicación de masas y cambio social. Ediciones Infinito. Buenos Aires. 2004.

Murdok, G. Proceso de cambio cultural. Instituto de Antropología Aplicada. Quito, Ecuador. s/f.

Boscardin. Lenguaje gráfico. Composición y ubicación de los elementos de diseño. www.perio.unlp.edu.ar/tpm.

Leone. Leyes de la Gestalt. www.guillermoleone.com.ar. 
